\title{
Preliminary Characterization of Thin Biofilms by Optical Microscopy
}

\author{
$\mathrm{M} \mathrm{C} \mathrm{CORTIZO}^{1}$ and M FERNÁNDEZ LORENZO DE MELE ${ }^{2 * *}$ \\ ${ }^{1}$ Cátedra de Materiales Dentales, Facultad de Odontología, Universidad Nacional de La Plata, 1900 La Plata, Argentina; \\ ${ }^{2}$ INIFTA, Facultad de Ciencias Exactas, Universidad Nacional de La Plata C C 16, Suc 4, 1900 La Plata, Argentina
}

(Received 27 September 1999; in final form 10 January 2000)

A simple non-invasive technique has been used that employs conventional optical microscopy and a glass flow cell to observe biofilms formed on opaque thin substrata. The technique allows the roughness of the biofilm and the substratum to be evaluated, and the biofilm thickness to be easily measured. The biofilm density may be quantified through colour gradients. In addition, some details of biofilm growth processes like the formation of water channels and pores, and interactions between planktonic and sessile cells can be visualized. Results related to the development of thin biofilms and their response to the environment under different conditions are reported. Pure and mixed microbial cultures and different solid substrata were assessed.

Keywords: microscopy; biofilm; bacteria; colonization; bacterial attachment; Pseudomonas fluorescens

\section{INTRODUCTION}

Most biofilms are composed of bacterial colonies embedded in extracellular polymeric substances (EPS) with open water channels between them.
Direct observation of biofilms is the "gold standard" because it allows observation of living, fully hydrated specimens in real time (Singleton et al., 1997). A complex architecture that includes microcolonies, water channel structures and "streamers" which oscillate and vibrate can be observed by high resolution techniques (Lewandowski, 1998; Stoodley et al., 1998). Although useful and widely applied, some of the high resolution techniques require extensive sample preparation ex situ (Beech et al., 1996). Electron microscopy has been used extensively for the examination of both surface structures and sectioned samples of biofilms (Newman \& Barber, 1995; Cortizo \& Mele, 1998). However, in the case of scanning electron microscopy (SEM), invasive pretreatment of the biofilm including dehydration is needed to preserve biological structures. Specimen distortion and introduction of artefacts may be found in some cases. Environmental scanning electron microscopy (ESEM) provides an useful tool which avoids the dehydration process because samples can be maintained at the saturated partial vapour pressure of water

\footnotetext{
*Corresponding author; e-mail: mmele@inifta.unlp.edu.ar
} 
at room temperature. Thus, examination of uncoated, non-conductive, wet samples is possible (Beech et al., 1996).

Recently, the introduction of confocal laser scanning microscopy (CLSM) has provided a step change in the capability for non-invasive imaging of biofilms. This technique allows the 3-D architecture of the biofilm to be assembled by gathering serial optical section images. Biofilm microstructure may be obtained by the application of appropriate image processing methods (Singleton et al., 1997). Atomic force microscopy (AFM) can also be used to examine biofilms in a non-invasive manner, and is more suited to the study of biological systems than scanning tunnelling microscopy (STM) because it offers greater operational flexibility (Beech et al., 1996).

Many laboratories do not have nanoscopes and CLSM routinely available. Thus, a simple and more accessible microscopic technique to follow the biofilm formation process has been developed. The growth of thin crystal layers can be followed with low resolution optical microscopy $(\times 5$ objective $)$ by a special assemblage (Azzaroni et al., 1999; Schilardi et al., 1999) and a similar procedure has been employed to follow the growth of thin biofilms. The design has been modified by using a higher magnification ( $\times 40$ objective) and a specially designed adapter.

The aim of the present paper is to demonstrate the use of conventional optical microscopy together with a glass flow cell to visualize biofilm microstructures (microcolonies, pores, channels) formed on thin opaque substrata, in real time. The technique allows the evaluation of the roughness, thickness and possibly the density of biofilms grown on thin substrata, before more complex high resolution techniques are employed. As examples, results related to the formation of biofilms under different conditions (with and without antimicrobial agents, on smooth or rough solid substrata) and their response to the environment are reported.

\section{MATERIALS AND METHODS}

\section{Substratum}

Sheets of different materials (silver, cellulose acetate, stainless steel, brass, glass and titanium) $5 \mathrm{~mm} \times 30 \mathrm{~mm}$ in size and between 0.05 to $0.10 \mathrm{~mm}$ in thickness were used as substrata. The formation of the biofilm on the lateral thin area $(0.05-0.10 \mathrm{~mm}$ height $\times 30 \mathrm{~mm}$ long) was observed by optical microscopy with a $\times 40$ objective. The metal sheets were polished with alumina $(1 \mu \mathrm{m})$, flamed and successively rinsed in ethyl alcohol and sterile water. Rough lateral surfaces (polished with 600 grade emery paper) and micro-thorny tips (used in dentistry to remove the nerve from the root canal) were also employed to study the effect of surface unevenness.

\section{Bacterial Culture Conditions and Harvesting}

Pure cultures of Pseudomonas fluorescens and a consortium collected from the oral cavity of several patients were used in the experiments. The strain of $P$. fluorescens employed in this work was isolated from a contaminated cutting oil emulsion and identified by biochemical tests. It was maintained in Cetrimide agar at $28^{\circ} \mathrm{C}$ and its purity was periodically checked by means of Gram stains, the oxidase test, and other biochemical tests and plating. The inoculum of P. fluorescens was prepared by suspending a Cetrimide agar slant $(24 \mathrm{~h}$ old) in $2 \mathrm{ml}$ of sterile culture medium. The culture medium (Barón et al., 1978) contained $\mathrm{NaCl} 6 \mathrm{gl}^{-1}, \mathrm{KCl} 0.3 \mathrm{gl}^{-1}, \mathrm{CaCl}_{2}$ $0.2 \mathrm{gl}^{-1}$, lactic acid $3.1 \mathrm{gl}^{-1}(\mathrm{pH}=6.5)$ with $0.5 \mathrm{gl}^{-1}$ yeast extract and 1 or $4 \mathrm{gl}^{-1}$ glucose. Experiments with the addition of $8 \mathrm{gl}^{-1} \mathrm{NaF}$ were also performed. All solutions were prepared from analytical grade chemicals. The inoculum was later poured into an Erlenmeyer flask containing $150 \mathrm{ml}$ of the culture medium and kept on a rotary shaker for $2 \mathrm{~h}$. 
The consortia used in the experiments were collected by scraping the gingival area of buccal and lingual tooth surfaces and along the entire fissure or margin of restorations on occlusal surfaces of several patients with a normal periodontal status. Each sample was dispersed by sonication for $10 \mathrm{~s}$ in sterile culture medium. Oral microorganisms were maintained both in TVLS medium (under anaerobic conditions) and brain heart $(\mathrm{BH})$ infusion. Every 2 months they were replaced by new samples obtained from the same patients. The inoculum was prepared by mixing equal quantities of the anaerobic and aerobic TVLS and BH media. An estimation of the planktonic cell number was made using a PetroffHauser camera. The inoculum was poured into an Erlenmeyer flask containing the culture medium. The initial number of cell was adjusted to ca $10^{5}$ cells $\mathrm{ml}^{-1}$.

\section{Microscopic Observations of the Biofilms}

A diagram showing the apparatus designed to follow biofilm growth including a glass flow cell adapted to the microscope and the light path is shown in Figure 1. Thin sheets of the different substrata were attached to cylinder-shaped stainless steel holders separated by $3 \mathrm{~mm}$ from the bottom of the glass cell. The glass cell was filled with the culture medium so that the sheets were covered. At appropriate time intervals biofilm development on the thin lateral area of the sheets was observed. The microscope objective was placed so that the biofilm/substratum interface

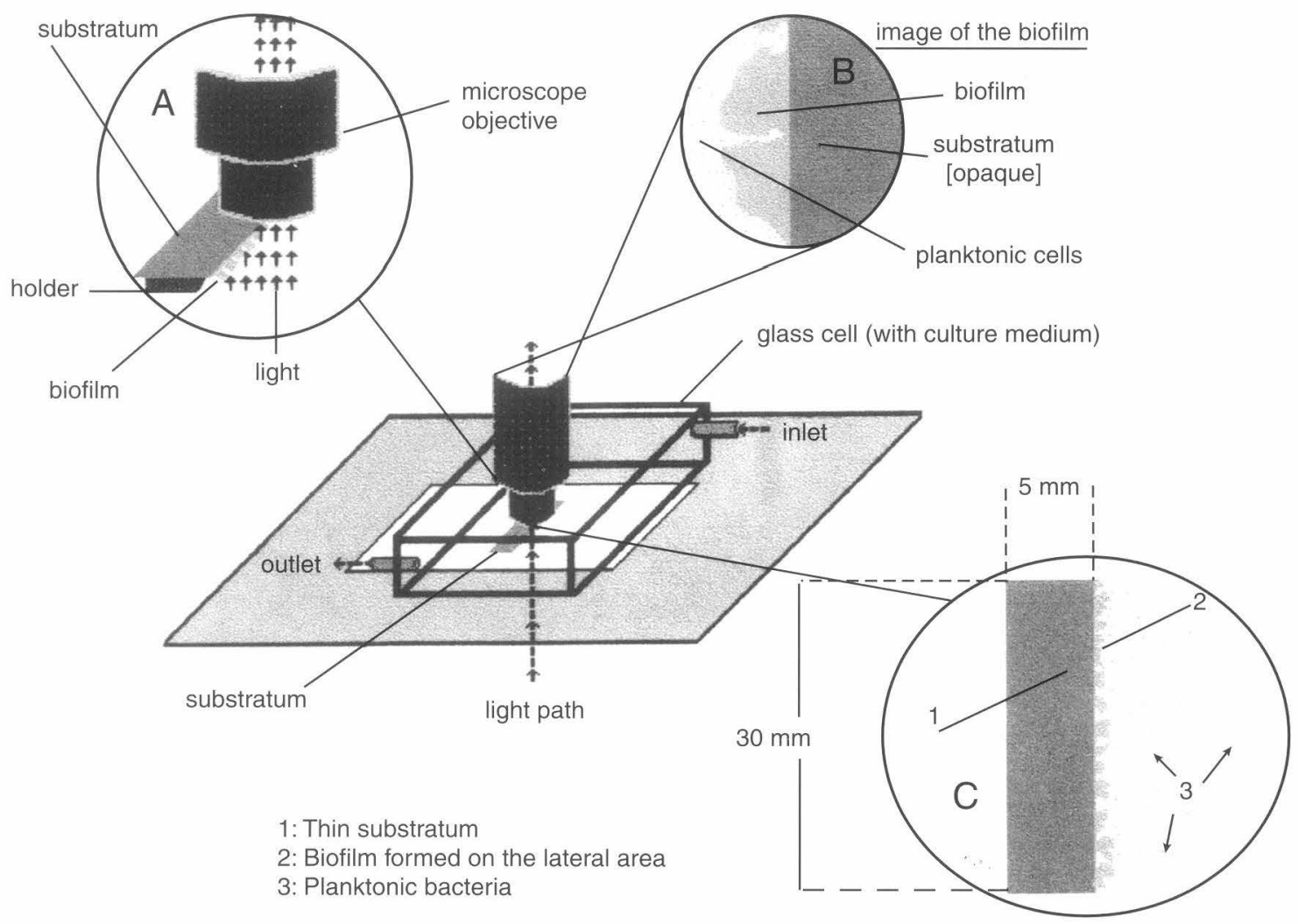

FIGURE 1 Diagram of the assembly used for microscopic observations of biofilms, including the light path and the flow cell ( $\mathrm{A}, \mathrm{B}$ and $\mathrm{C}=$ details). 
was in the middle of the field (see Figure 1, insets $A$ and B). The substratum was visualized as an opaque surface and the biofilm and the bathing solution as a bright area using a $\times 40$ objective.

\section{RESULTS AND DISCUSSION}

Continuous optical microscopy of biofilms grown on thin substrata (OMBT) was performed. The different processes which give rise to the formation of the biofilm were observed, viz. a) transport of cells from the bulk towards the substratum/solution interface, b) attachment of cells to the substratum surface, c) growth of cells already attached to the surface, and d) adhesion of cells to the biomass already attached to the surface. Figure 2 shows the early stages of the development of two colonies of the consortium of oral microorganisms. The formation of biomass aggregates may indicate a role for inter- and intraspecies adhesion mechanisms during colonization (Singleton et al., 1997). It was observed that the size and shape of the colonies was dependent on glucose concentration and on the microorganisms and substratum involved. Higher rates of accumulation of EPS and bacteria were detected

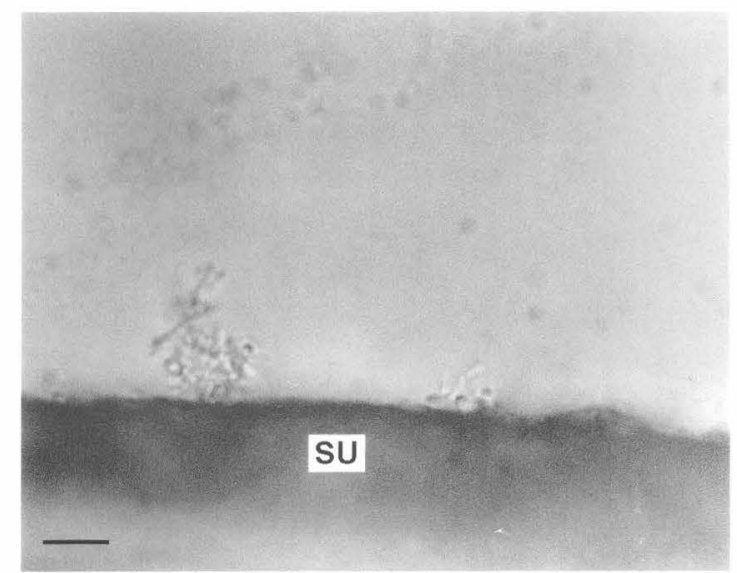

FIGURE 2 Optical photomicrograph showing the formation of two colonies of the consortium of oral microorganisms, on a cellulose acetate sheet after $40 \mathrm{~min}$ exposure. Bar $=70 \mu \mathrm{m} ; \mathrm{SU}=$ substratum. (See Color Section.) at higher glucose concentrations under static conditions. The addition of an antimicrobial agent such as fluoride to the liquid medium resulted in a darker inner layer with lower transparency (Figure 3). This colour gradient can also be seen in Figure 4 and seems to be related to an EPS density gradient (Mele \& Cortizo, 2000).

After observing the development of the biofilm by OMBT for several hours it was observed that the motility of $P$. fluorescens and the environmental transformation of the interface by the cells already attached, played important roles in the attachment process. Close to the $24 \mathrm{~h}$ old $P$. fluorescens biofilm/solution interface, a "viscous" layer could be visualized by careful examination of the field when the flow rate was increased. The velocity of small inert particles present in the medium and that of planktonic cells was lower in this region. The arrow in Figure 4 indicates the viscous area

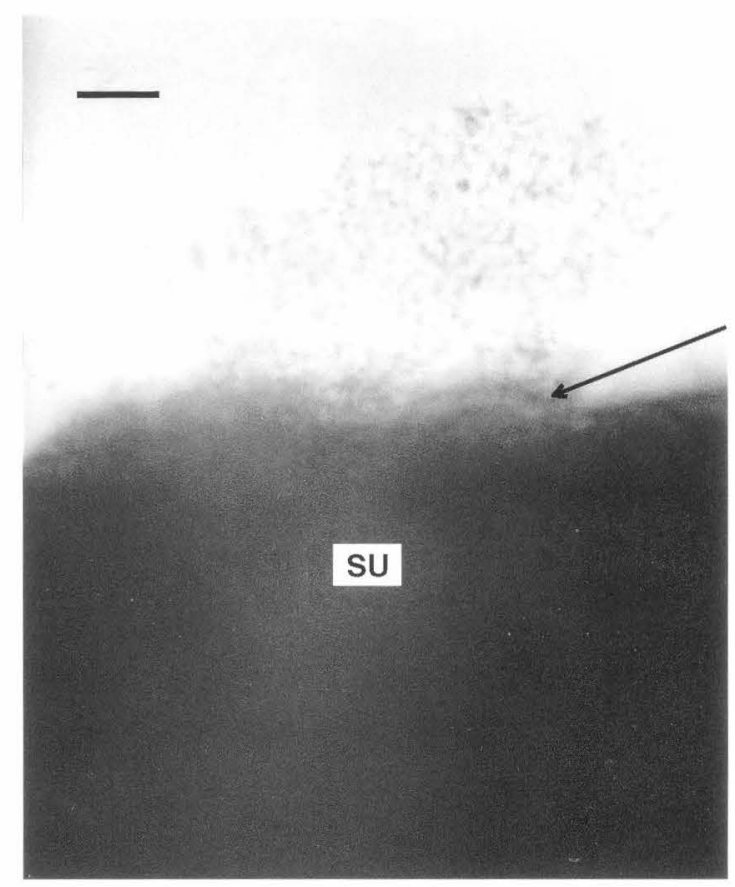

FIGURE 3 Optical photomicrograph showing a colony of the consortium of oral microorganisms formed on a stainless steel sheet immersed in fluoride-containing saliva $\left(8 \mathrm{gl}^{-1}\right)$ after $4 \mathrm{~d}$ exposure. The arrow indicates the more compact inner layers. Bar $=70 \mu \mathrm{m} ; \mathrm{SU}=$ substratum. (See Color Section.) 


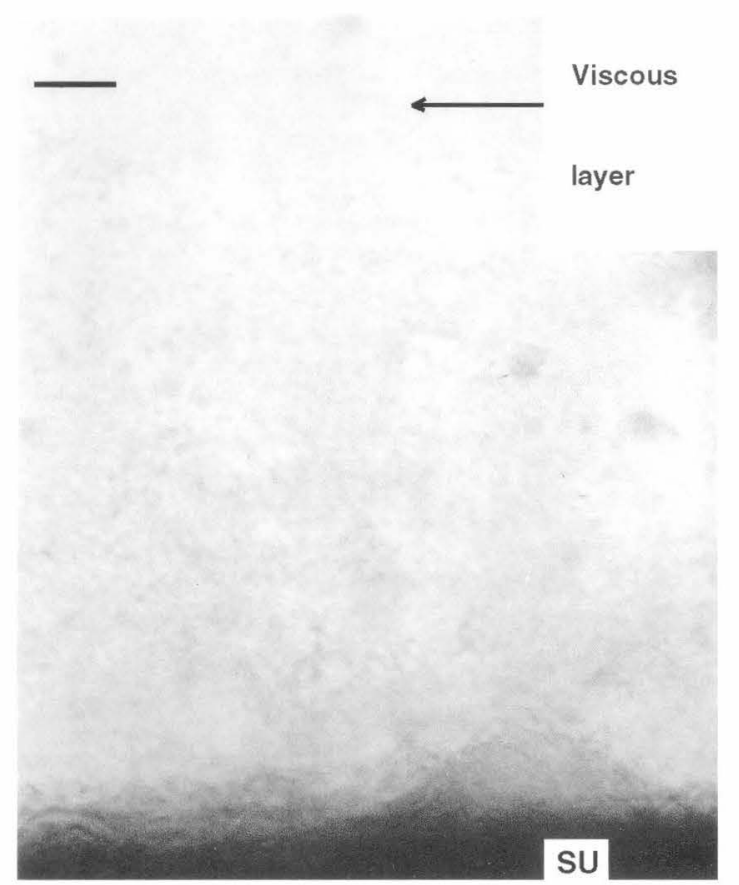

FIGURE 4 Optical photomicrograph showing a $3 \mathrm{~d}$ old $P$. fluorescens biofilm formed on a brass sheet. The colour gradient appears to be related to a biofilm density gradient. The arrow shows the viscous liquid layer. $\mathrm{Bar}=70 \mu \mathrm{m}$ $\mathrm{SU}=$ substratum. (See Color Section.)

which could be visualized easily by direct microscopic observation under flow. The thickness of this layer varied between a few and several hundred micrometers.

Microscopic observations carried out during biofilm formation are worthy of further investigation. Firstly, there was not a random movement of P. fluorescens planktonic cells but a preferential displacement towards the biofilm/solution interface, combined with a tendency for grouping of the planktonic cells. In addition, there was a particular motion of planktonic bacteria around the border of the colonies and a higher planktonic bacterial density in this region was observed compared to that around the uncolonized substratum or in the bulk solution. Sessile cells attached to the border of the colonies appeared to interact with the planktonic bacteria (Singleton et al., 1997; Davies et al., 1998; Costerton et al.,
1999). It was concluded that the behaviour of these bacteria during colony formation is very different from that of colloidal particles during aggregation processes. Thus, the use of theories that strictly define bacteria as colloidal particles is inappropriate in this case.

Microbial attachment and microcolony formation to a valley of a micro-thorny tip was also observed by OMBT over a $24 \mathrm{~h}$ period (Figure $5 \mathrm{a}$ and $5 \mathrm{~b}$ ). The motility of $P$. fluorescens cells facilitated the attachment of the cells inside the grooves (Scheuerman et al., 1998).

Details of the complex architecture of biofilms such as interstitial channels and pores within microcolonies, and water channels between clusters were also visualized by OMBT (Figure 6). Water channels were frequently formed under the biofilm/solution interface when neighbouring colonies coalesced at the upper side, close to the solution, while the inner side, close to the substratum, remained separated (Beyenal et al., 1998; Rasmussen \& Lewandowski, 1998) Channel depth evaluation was limited to the depth of field of the microscope objective and it was useful to take photographs at different focal planes. Colour and transparency gradients appeared to be related to biofilm density gradients. A darker colour and less transparency in the inner older layer than in the outer younger layer were detected in $P$. fluorescens colonies in stationary phase growth (Figures 4 and 6). It appears that there are higher numbers of cells per unit area in the darker region of Figure 6.

Roughness of the biofilm and the substratum were evaluated by OMBT (Figure 7). A straight front of growth at the biofilm solution interface was formed (see arrows in Figure 7). The biofilm thickness was not uniform and varied to obliterate the unevenness of the surface, in agreement with previous SEM observations (Mele \& Cortizo, 2000). The biofilm thickness can be easily measured using the OMBT technique.

A limitation of the OMBT technique is that hydrodynamic conditions are undefined in the region where the biofilm is observed, including 

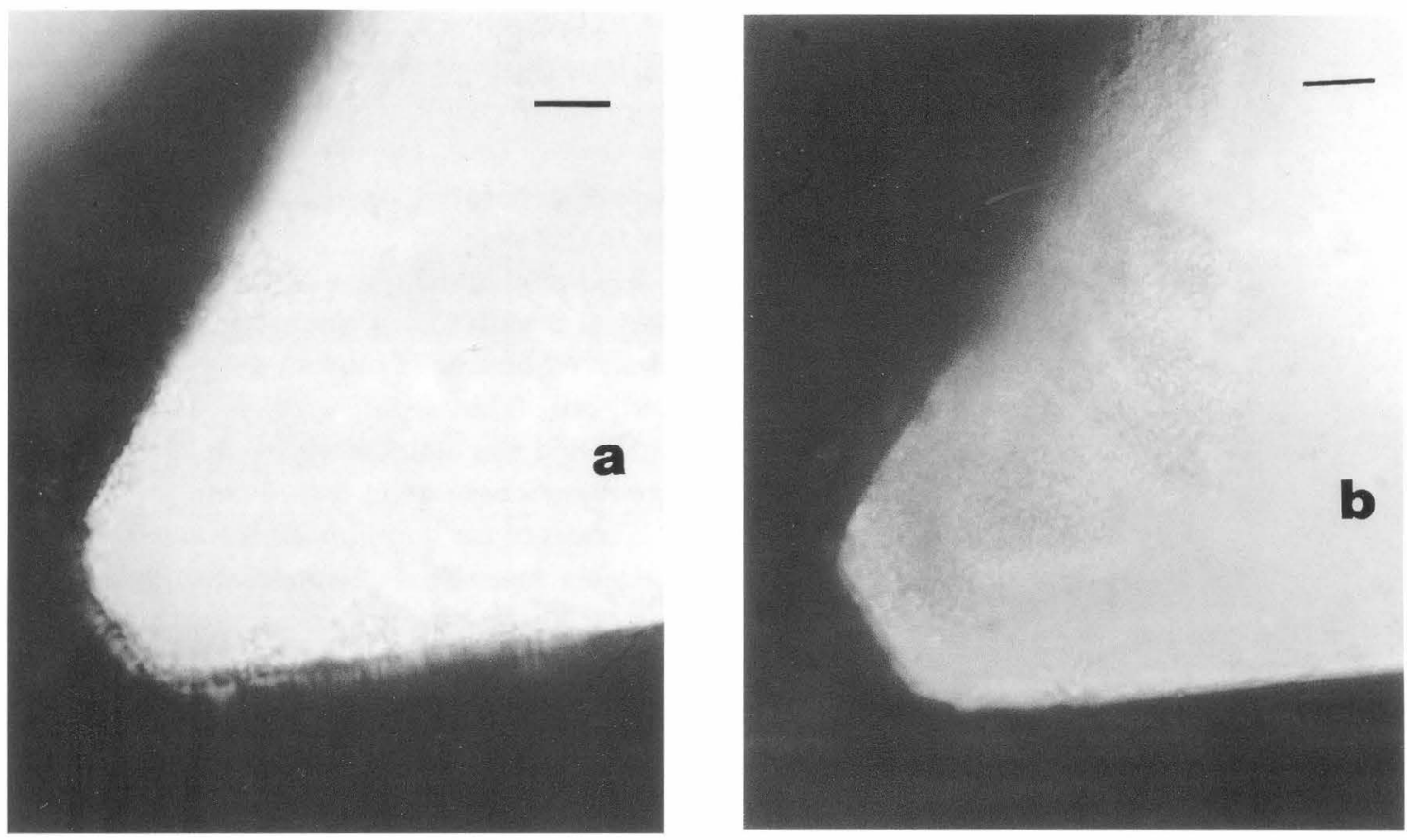

FIGURE 5 Optical photomicrograph showing two different stages of a $P$. fluorescens biofilm formed on a micro-thorny tip. $\mathrm{a}=$ after $4 \mathrm{~d}$ immersion; $\mathrm{b}=$ after $6 \mathrm{~d}$ immersion; $\mathrm{bar}=91 \mu \mathrm{m}$. (See Color Section.)

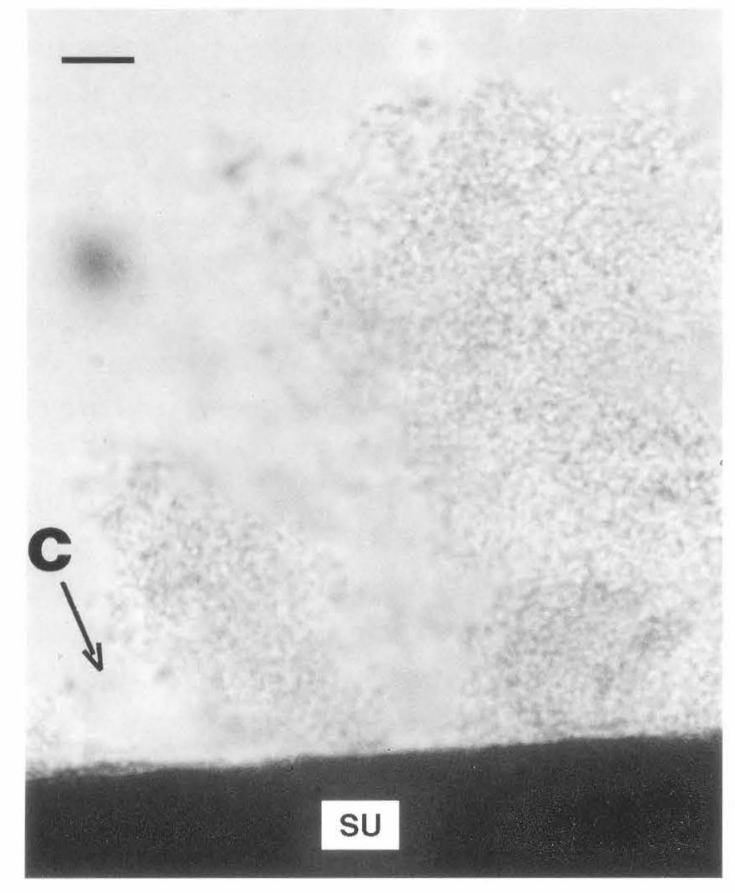

FIGURE 6 Optical photomicrograph showing channels (c) in a $6 \mathrm{~d} P$. fluorescens biofilm formed on a silver sheet. $\mathrm{Bar}=91 \mu \mathrm{m} ; \mathrm{SU}=$ substratum. (See Color Section.)

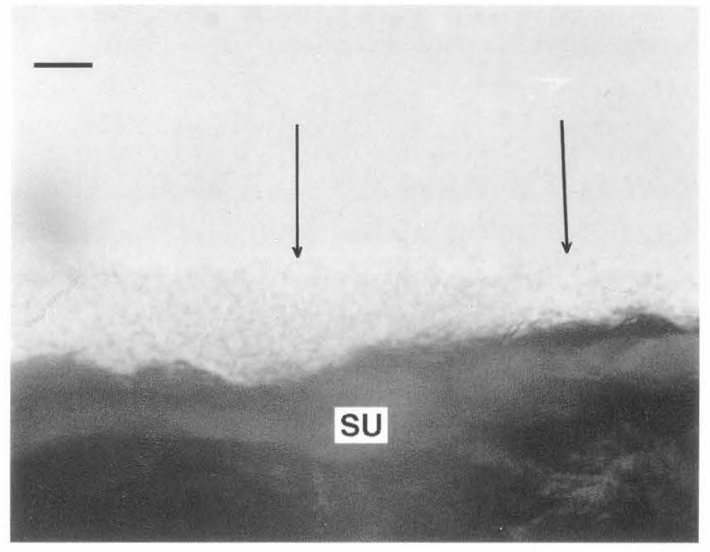

FIGURE 7 Optical photomicrograph showing a biofilm covering a rough brass sheet after $2 \mathrm{~h}$ exposure. The thickness of the biofilm varies forming a straight horizontal front of growth at the biofilm/solution interface (arrows). Bar $=70 \mu \mathrm{m}$; SU $=$ substratum. (See Color Section.)

edge effects. However biofilm movements could be observed under flow conditions, and these were slower than those of the bulk because of their elastic characteristics and the viscous nature of the surrounding liquid layer. Sometimes 


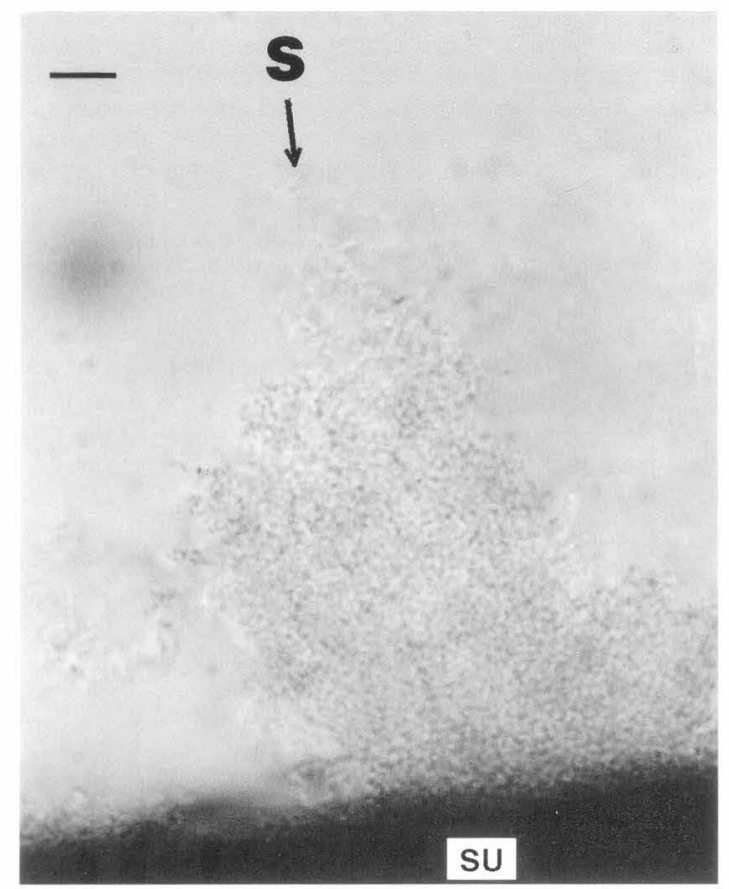

FIGURE 8 Optical photomicrograph showing a $7 \mathrm{~d}$ $P$. fluorescens colony on a silver sheet with a streamer (S) at the top. Darker regions appear to correspond to a higher biofilm density in this area. $\mathrm{Bar}=70 \mu \mathrm{m}$; SU = substratum. (See Color Section.)

"streamers" (Stoodley et al., 1999) of bacteria embedded in EPS (Figure 8) could be seen stretching, relaxing and oscillating. Oscillations under static conditions were also detected.

\section{CONCLUSIONS}

The OMBT technique employed conventional optical microscopy together with a flow cell and opaque thin substrata to visualize biofilm microstructure and to evaluate the roughness of the substratum and the thickness of biofilms. Limitations were related to the undefined hydrodynamics in the region where the biofilm was observed, including the influence of edge effects, and the evaluation of channels and pore depths was restricted by the depth of the field of the microscope.
Motile P. fluorescens cells colonised grooves forming an even biofilm over rough substrata. Motility played an important role during colony formation and the behaviour of the motile cells was very different from that of colloids during an aggregation process. Thus, care must be taken when such microorganisms are defined as colloids in theoretical simulations.

\section{Acknowledgements}

The authors thank Dr P Schilardi and R C Salvarezza for their helpful advise on the microscopic observation procedures. This research work was supported by the University of La Plata and by the National Council for Scientific and Technical Research (CONICET).

\section{References}

Azzaroni O, Schilardi P L, Salvarezza R C, Arvia A J (1999) Smoothening mechanism of thiourea on silver electrodeposition. Real time imaging of the growth front evolution. Langmuir 15: 1508-1514

Baron C, Marschoff C M, Aragon P J (1978) Corrosion rates of dental amalgam in Ringer solutions and artificial saliva. J Life Sci 8: 105-110

Beech I B, Cheung C W, Johnson D, Smith J R (1996) Comparative studies of bacterial biofilms on steel surfaces using atomic force microscopy and environmental scanning electron microscopy. Biofouling 10: 65-77

Beyenal H, Tanyolac A, Lewandowski Z (1998) Measurement of local effective diffusivity in heterogeneous biofilms. Water Sci Technol 38: 171-178

Cortizo M C, Mele, M F L de (1998) Biofilm formation on dental metallic materials. Effect of fluoride anions. In: Gaylarde C C, Barbosa T C P, Gabilan N H (eds) Proc 3rd Latin American Symp Biodeterioration and Biodegradation. Br Phycol Soc, UK, Paper No 18

Costerton J W, Stewart P S, Greenberg E P (1999) Bacterial biofilms: a common cause of persistent infections. Science 284: $1318-1322$.

Davies D G, Parsek M R, Pearson J P, Iglewski B H, Costerton J W, Greenberg, E P (1998) The involvement of cell-to-cell signals in the development of a bacterial biofilm. Science 280: 295-298

Lewandowski Z (1998) Structures and function of bacterial biofilms. Corrosion'98, Paper No 296. NACE International, Houston, TX

Mele M F L de, Cortizo M C (2000) Biodeterioration of dental materials: influence of bacterial adherence. Biofouling 14: 305-316

Newman H M, Barber P M (1995) Dental plaque structure and biology. In: Wimpenny J, Handley P, Gilbert P, Lappin-Scott H (eds) Life and Death of Biofilm. Bioline, Cardiff, UK, pp 27-32 
Rasmussen K, Lewandowski Z (1998) Microelectrode measurements of local mass transport rates in heterogeneous biofilms. Biotechnol Bioeng 59: 302-309

Singleton S, Treloar R, Warren P, Watson G K, Hodgson R, Allison C (1997) Methods for microscope characterisation of oral biofilms: analysis of colonisation, microstructure and molecular transport phenomena. Adv Dent Res 11: 133-149

Scheuerman T R, Camper A K, Hamilton M A (1998) Effects of substratum topography on bacterial adhesion. J Colloid Interface Sci 208: 23-33
Schilardi P L, Azzaroni O, Salvarezza R C, Arvia A J (1999) The validity of Kardar-Parisi Zhang equation in the asymptotic limit of metal electrodeposition. Phys Rev 859: 4638-4641

Stoodley P, Lewandowski Z, Boyle J D, Lappin-Scott H M (1998) Oscillation characteristics of biofilm streamers in turbulent flowing water as related to drag and pressure drop. Biotechnol Bioeng 57: 536-544

Stoodley P, Dodds I, Boyle J D, Lappin-Scott H M (1999) Influence of hydrodynamics and nutrients on biofilm structure. J Appl Microbiol 85: 19S-28S 